\title{
SURVEY SuRfER: A WEB BASED DATA GATHERING AND ANALYSIS APPLICATION
}

\author{
Mrs. Dhanamma Jagli ${ }^{1}$, Ms. Krutika Tawde ${ }^{2}$, Mrs. Pratima Musale ${ }^{3}$, \\ Ms. Swathi Muthukumar ${ }^{4}$ \\ ${ }^{1}$ Department of MCA,V.E.S.Institute of Technology, Mumbai, India \\ ${ }^{2,3,4}$ MCA Final year Students, V.E.S. Institute of Technology, Mumbai, India
}

\begin{abstract}
The most important need for a web based survey technology is speedy performance and accurate results .Though there are variety of ways a survey can be taken manually and have it assessed manually but here we are introducing the survey site concept where the assessment of the survey responses are created automatically by applying certain statistics. In this paper we propose the idea of automated approach to the analysis of the survey where the results would be evident graphically to take a wise decision. Additional to graphical view we aim on forming a platform to view complex, tedious data in simple, understandable, interactive and visualized form.
\end{abstract}

\section{KEYWORDS}

Data Analysis, Data Mining, Real Time Analysis, Three Tier Architecture, Web Based Survey.

\section{INTRODUCTION}

This paper presents Survey Surfer, a Web-based survey development and implementation technology, designed for creating on-line surveys for generic purposes. Surveys are considered as general data collection field of application which provide the survey creator on-line access to real time information on survey responses. The system is a three-tiered database-backed application that has been developed to support the user to gain insights for easy decision making. Surveys are mostly taken manually. Mostly the techniques used to analyse these gained information have to undergo much manual processing and there is a need of expert advice to take decisions. Analysis of the data obtained from gaining the responses from the visitor to the sites are visually displayed with the help of Ms charting controls. This tool is helpful in giving the output into different graph types and thereby helping to compare the results in many different views and perspective, providing insights to the user to reach to a certain decision.

This technology provides data mining process for exploring data in search of consistent pattern by using real time analysis over the received data.

\section{RELATED WORK}

The main purpose of Survey Surfer application is to interact with user as well as present data in well-defined style and matter. Robert A. Dennis and Sanjiv S. Gambhir [2] have proposed an approach to move survey-building software application toward the WWW by exporting content to HTML format [6][7].Addition to usage of HTML and ASP.NET server technology, dynamic branching is possible using custom set of interfaces where response to the next page is already set to default values. When the respondents answer is not accurate with the default response answers then the next page is invoked only when none of the defined response are encountered. 
Robert A. Dennis and Sanjiv S. Gambhir [2] developed iQ\&A system where questions are added under a particular section and creator of survey can edit the changes to those sections even after survey creation. The order of questions within a particular sections and the sections of the survey are sorted by the use of a unique graphical sorting tool. Whereas in the Survey Surfer application, Excel sheet is provided for creating the questionnaires with appropriate validation. Data Visualisation and its analysis is made possible with the help of MS chart controls, which is compatible with the .Net Framework as shown in fig 5[1]. The Survey Surfer application provides the drill down approach for visualization of data in Admin Dashboard as shown in fig 2[7].

Sebastian Lauer and Mihai Lazarescu [1] proposed a visual data analysis tool developed for comparison and change detection of the sport player's performance. Where previous conditions and minimum norms and parameters of players are considered, which are compared with the real time statistics. This comparison results in the change detection and helps in gaining insights and estimation about the player's future performance.

\section{Proposed System}

\subsection{THREE TIER ARCHITECTURE}

The basic concept of a tiered architecture involves breaking up an application into logical chunks, or tiers, each of which is assigned general or specific roles. We have implemented the 3-tier architecture to gain high data structure security, where each and every query is written as a stored procedure. The stored procedure is pre compiled object which helps in reducing the query execution time and reduces the risk of security being breached by SQL injection as no table name or query entries exist in the aspx or aspx.vb page.

The architecture of the whole system is as in Fig (1) where the first application layer contains the aspx and the code behind it i.e. aspx.vb pages while the DAL(Data Access Layer) have the common query procedures used to access the database. Data access layer acts as a gateway between the application layer [.asp page (presentation) \& .aspx.vb page (business logic)] and the MySQL database.

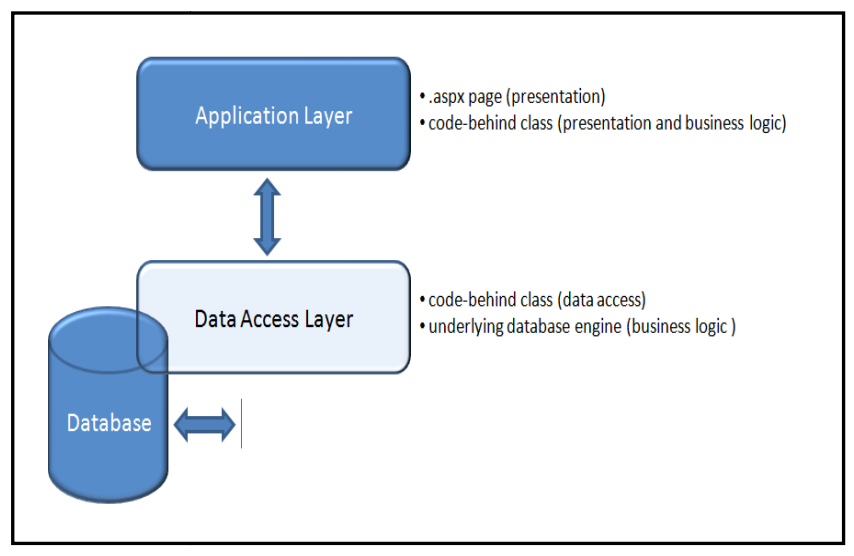

Figure 1. 3-Tier Architecture.

\subsection{DATA Mining}

Data mining is the process of examining the data obtained and gain new insights from those. It allows users to analyse data obtained from the survey taken by the visitors, visualize them in 
different perspectives by changing the dimensions of charts and summarize the results. Technically, data mining is the process of finding correlations or patterns among dozens of fields in large relational databases.

In information technology, to move from summary information to detailed data by focusing in on something, drill-down is used. Drill-down analysis is one of the most common forms of operations carried out in the survey site analytics, and the term is commonly heard in game data mining contexts[10]. This is because drill-down analysis is a very comprehensible method for indepth analysis of data, making it very useful for the behaviour analysis of users and visitors, where the root causes of behavioural patterns are often nestled deep within the data.

The dashboard shows administrator the view for all information about website such as total no of users, total no of surveys, total no of visitors, through a drill down perspective as shown in the Figure 2(a), 2(b) and 2(c)[7]. Where the Figure 2(a) represents the total number of users joined the Survey Surfer site on yearly basis, when clicking on the particular bar of the year it drills down to the required part on quarterly basis as displayed in Figure 2(b) which can be further reduced to the monthly basis level as shown in Figure2(c). By this a higher level of abstraction is materialized by consolidating the metrics at a lower level.

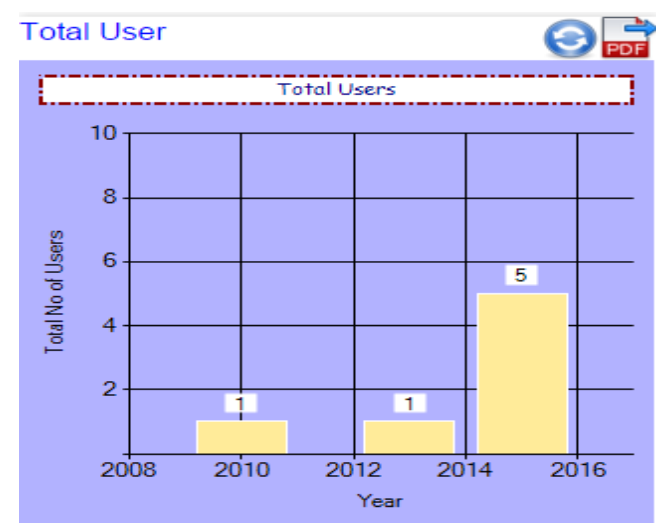

Figure 2(a). Total No of Users (yearly).

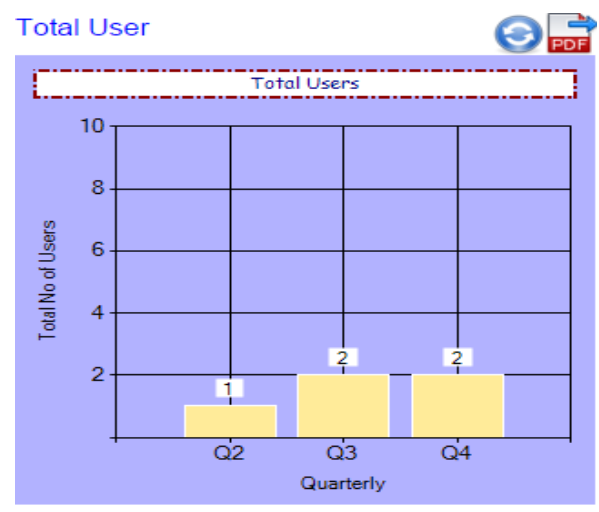

Figure 2(b). Total No of Users (Quarterly). 


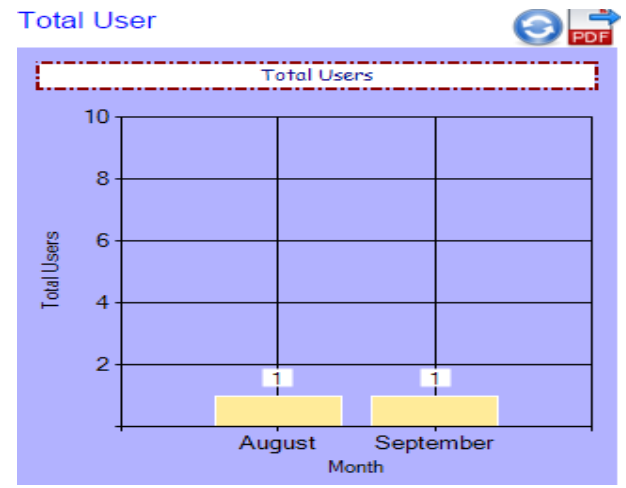

Figure 2(c). Total No of Users (Monthly).

\subsection{General Workflow Of Web BaSed Survey Site}

The web based survey site provides services for creating customized online surveys, distributing them via the internet through email, and tabulating results automatically in real time. Figure (3) explains the work flow of the web based survey.

The site has 3 profiles - Admin, Users and Visitors. Visitors are the one who take surveys created by the Users. The surveys could be created in any of the categories provided. Users can create survey and based on the responses received for the survey, gain insights to make decision based on the data shown through graphical visualization. The surveys could be made available to all the visitors publicly or can be made private by selecting the visitors and mailing the details only to selected visitors. Based on the feedback given by the visitors, users are able to extract the overall impact of their product/service in the masses. Admin is able to visualize the activities going within the site in terms of number of users, visitors and surveys over the period of time.

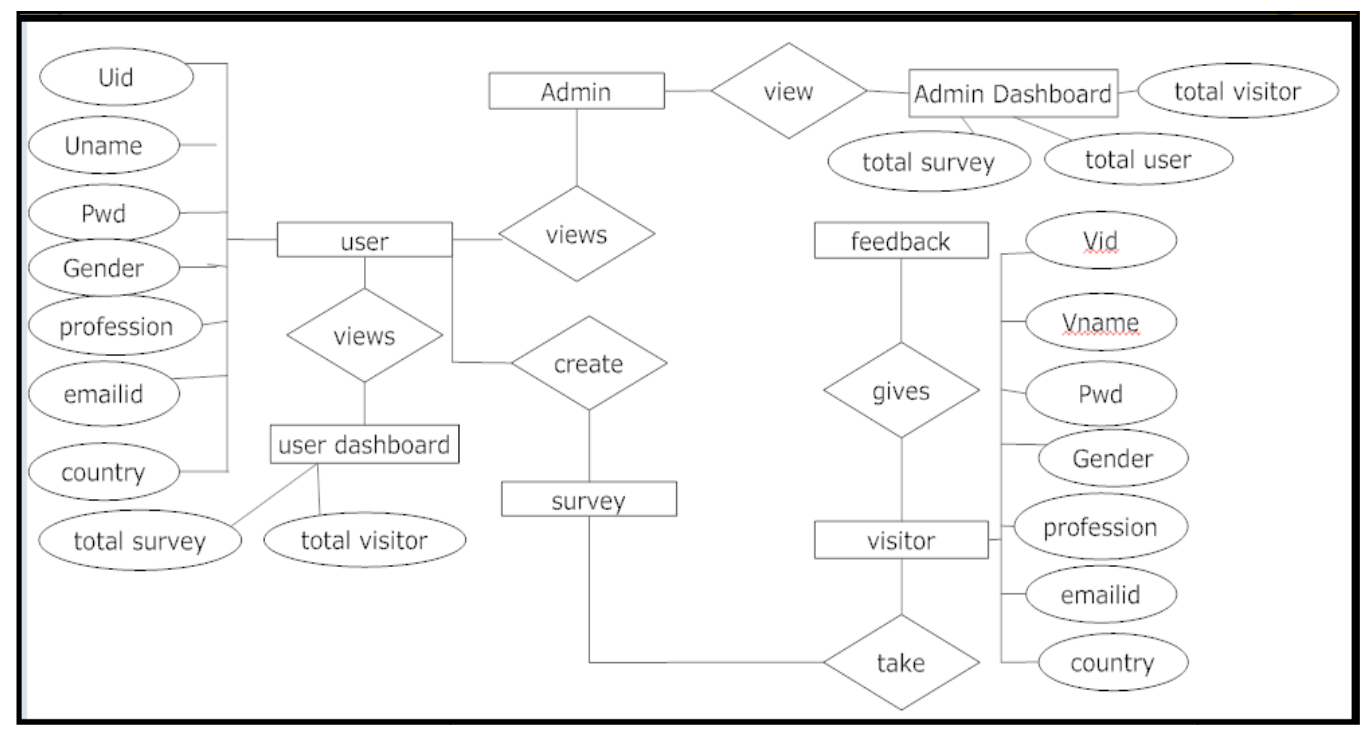

Figure 3. Work Flow of Web Based Survey. 


\section{Methodology Of Data Analysis}

The data analysis is done according to the survey title and each survey has its own certain attributes of criteria based on which the analysis parameters are set, which are as follows:

1. Country

2. State

3. City

4. Gender

5. Age

6. Question

Figure (4) shows us the charts generated dynamically based on the selected criteria.
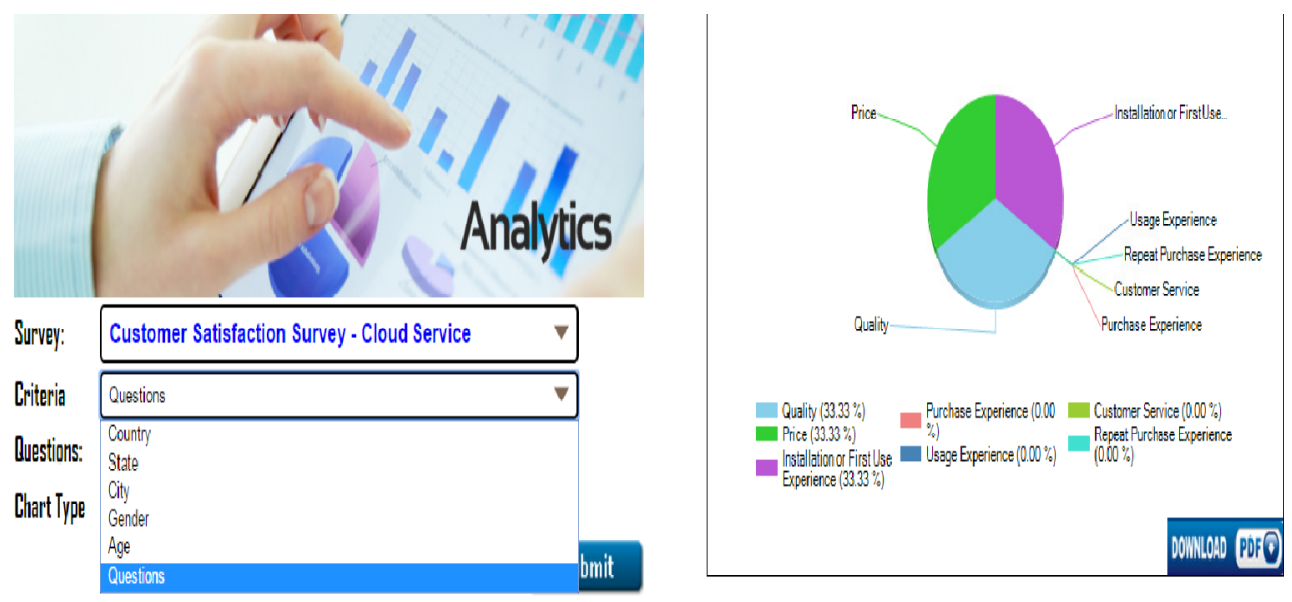

Figure 4. Criteria's involved for analysis.

There are varied variety of charts like the one shown in figure 5(a),5(b),5(c),5(d) that can be visualised through which the user is able to have different aspects to compare them with . Hence, making way to a good plan or an appropriate decisions.

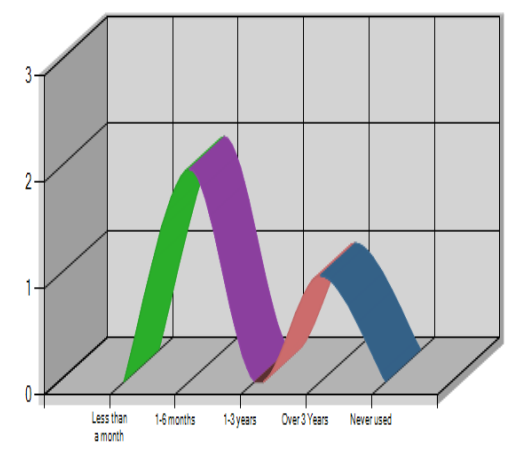

Figure 5 (a). Spline chart.

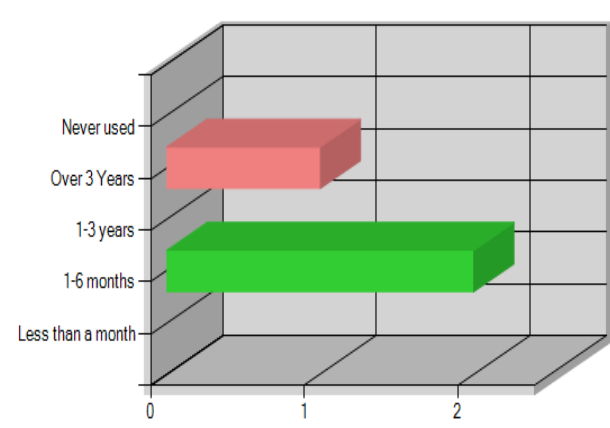

Figure 5(b). Stacked bar chart. 


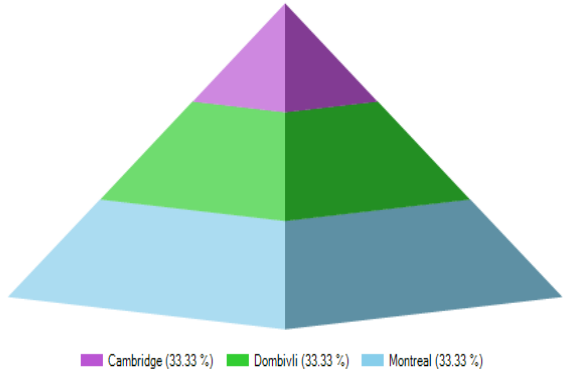

Figure 5(c). Pyramid chart.

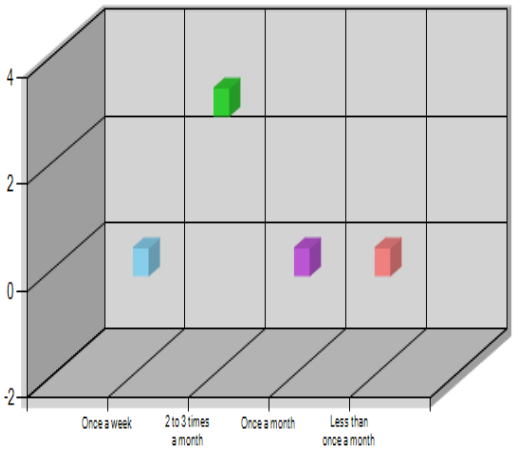

Figure 5(d). Bubble chart.

Data Visualisation and its analysis is made possible with the help of MS chart controls, which is compatible with the .Net Framework. The MS chart controls helps to create ASP.NET pages with uncomplicated, comprehensible and visually efficacious charts for real time data analysis.

It also provides the dashboard for the Administrator through which he is able to view all the information about website such as total no of users, total no of surveys, total no of visitors, through a drill down perspective as shown in the Figure 2(a),2(b) and 2(c). Geo chart is used, which helps in analysing the concentration of users and visitors using the site geographically based on their location .Figure (6) shows the geographical mapping of the number of survey created country wise.

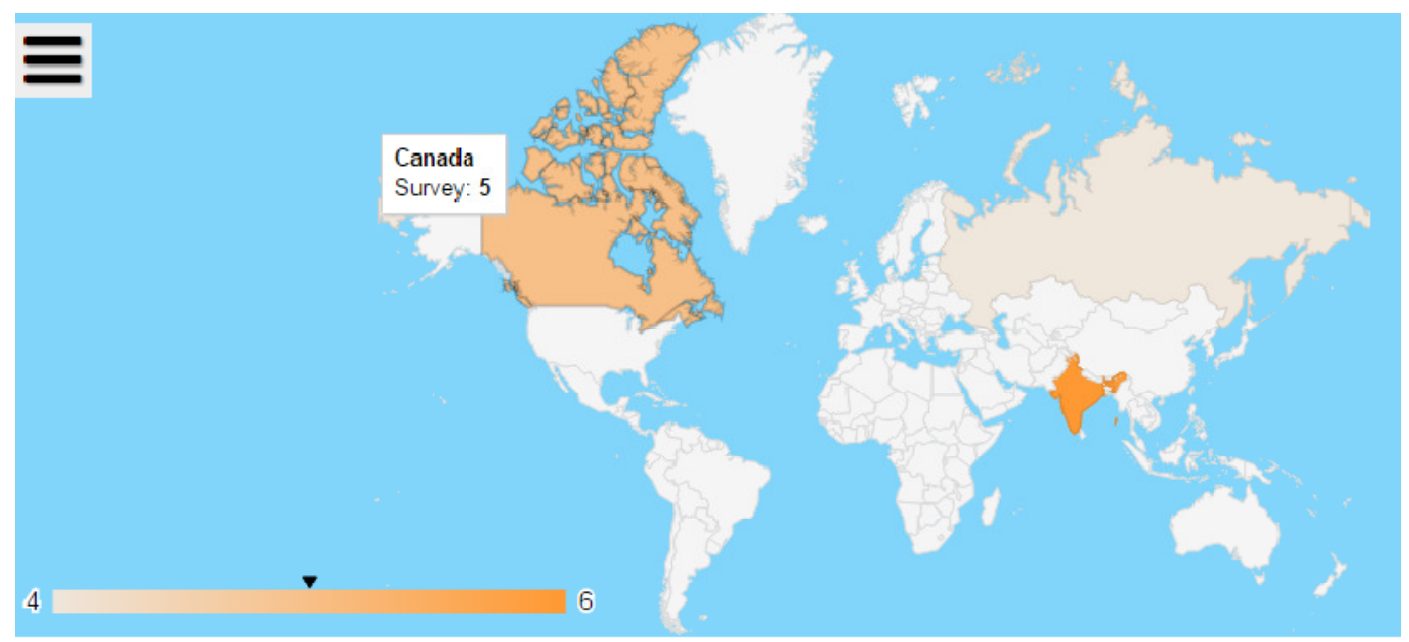

Figure 6. Geo Chart showing geographical mapping of number of survey created country wise

\section{Conclusions}

We have developed a web-based survey technology with potential for generic usage .The simplicity of survey creation and publishing, notification via email support, access to published surveys with an Internet browser, and powerful real time reporting capabilities provide a unique environment, which it is easy to construct, administer, and analyse surveys for various applications .Continued refinement of Survey Surfer should lead to an even more powerful system with a large array of applications 
Advanced Computational Intelligence: An International Journal (ACII), Vol.3, No.3, July 2016

\section{REFERENCES}

[1] Sebastian Lahr and Mihai Lazarescu ,"A Visual Data Analysis Tool for Sport Player Performance Benchmarking,Comparison and Change Detection", 19th IEEE International Conference on Tools with Artificial Intelligence

[2] Robert A. Dennis and Sanjiv S. GambhirJ. Breckling, Ed.,Internet Question \& answer(iQ\&A):A web based survey technology. Berlin, Germany: Springer, 1989, vol. 61.

[3] Robert A. Dennis and Sanjiv S. GambhirJ. Breckling, Ed., The Analysis of Directional Time Series: Applications to Wind Speed and Direction, ser. Lecture Notes in Statistics. Berlin, Germany: Springer, 1989, vol. 61.

[4] www.wikipedia.org

[6] Extracting and Visualizing Insights from Real-Time Conversations Around Public Presentations Nicolas Garcia Belmonte Visual Analytics Science and Technology (VAST), 2014 IEEE Conference on 25-31 Oct. 2014

[7] Anil Singh, Aakash Taneja, and George Mangalraj, "Creating Online Surveys: Some Wisdom from the Trenches Tutorial", IEEE transactions on professional communication, vol. 52, No. 2, June 2009.

[8] Ronald J. Glotzbach, Laura A. Kocur," Work in Progress - Evaluation and Feedback for Web Programming Curriculum ", October 12 - 15, 2011, Rapid City, SD 41st ASEE/IEEE Frontiers in Education Conference Session F4E.

[9] http://techbrij.com/drill-down-asp-net-chart-control, Drill down for ASP.NET 4.0 Chart Control

[10] http://blog.gameanalytics.com/blog/using-drill-down-analysis-to-find-out-why.html, Using drill-down analysis to find-out why.

\section{Authors}

\section{Mrs.Dhanamma Jagli}

Short Biography: Mrs.Dhanamma Jagli is an Assistance professor in V.E.S Institute of Technology, Mumbai, currently Pursuing Ph.D in Computer Science and Engineering and received M.Tech in Information Technology from Jawaharlal Nehru Technological University, Hyderabad and Andhra Pradesh. She has around 12 years teaching experience at the post Graduate and undergraduate level. She had published and presented papers in refereed

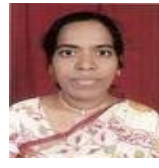
international journals and conferences. Her areas of research interest are Data Mining, Cloud Computing, Software Engineering, Database Systems and Embedded Real time systems. She has been associated with Indian Society of Technical Education (ISTE) as a life member.

\section{Ms. Krutika Tawde}

Short Biography:Krutika Tawde is currently a final year MCA student in Vivekanand Education Society's Institute of Technology (V.E.S.I.T), Mumbai. She has experience in software development and maintenance using .NET and SQL technologies of about a year and a half. A technology enthusiast and avid reader, she is interested in open source systems, data mining and analysis as well as application development.

\section{Ms. Pratima Musale}

Short Biography:Pratima Musale has completed her BCA from C.O.C.S.I.T. Latur, from S.R.T. University, Nanded. She is currently pursuing her MCA from Vivekanand Education Society's Institute of Technology, Mumbai. She has an abiding interest in Software Engineering and programming languages like $\mathrm{C}, \mathrm{C}++$.

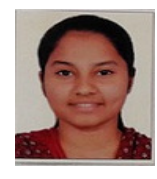

\section{Ms. Swathi Muthukumar}

Short Biography:Swathi Muthukumar is a final year student of Master of Computer Application (M.C.A) from Vivekanand Education Society's Institute of Technology (V.E.S.I.T), Mumbai University. Swathi has completed her B.Sc. In Computer science from Ramniranjan Jhunjhunwalla College, Mumbai University. She has been selected as an intern at Nomura. She is an ardent programmer with an abiding interest in data analytics and programming languages

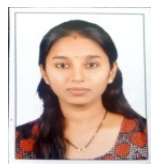
like $\mathrm{C}, \mathrm{C}++$ and. Net technologies. 